\title{
Pattern and predictors of dual antiplatelet use after coronary artery bypass graft surgery
}

\author{
Makoto Mori, MD, ${ }^{\mathrm{a}}$ Kayoko Shioda, DVM, MPH, ${ }^{\mathrm{b}}$ James J. Yun, MD, PhD, ${ }^{\mathrm{a}}$ Abeel A. Mangi, MD, MBA, ${ }^{\mathrm{a}}$ \\ Umer Darr, MD, ${ }^{a}$ and Arnar Geirsson, MD ${ }^{\mathrm{a}}$
}

\section{ABSTRACT}

Background: Resumption of dual antiplatelet therapy after coronary artery bypass grafting in patients presenting with acute coronary syndrome is recommended, but the current practice pattern in the United States remains unknown. We aimed to investigate the current pattern of dual antiplatelet therapy use after coronary artery bypass grafting at the Yale-New Haven Hospital.

Methods: We conducted a single-center retrospective review of patients who presented with acute coronary syndrome and underwent coronary artery bypass grafting between 2014 and 2016. The primary outcome was hospital discharge with dual antiplatelet therapy. Mixed-effect multivariate logistic regression was used to evaluate predictors of dual antiplatelet therapy use or nonuse, accounting for surgeon-specific preference. The discriminatory ability of the model was evaluated with receiver operating characteristics analysis.

Results: Of 572 patients included, only $29 \%$ were discharged with dual antiplatelet therapy. In the mixed-effect multivariate model isolating surgeon preferences, increase in age (odds ratio, $0.95 ; 95 \%$ confidence interval, $0.92-0.98 ; P<.001$ ) and discharge with anticoagulants (odds ratio, $0.20 ; 95 \%$ confidence interval, 0.07-0.55; $P=.002$ ) were associated with lower odds of dual antiplatelet therapy use. Off-pump coronary artery bypass grafting compared with on-pump coronary artery bypass grafting was associated with increased odds of dual antiplatelet therapy use (odds ratio, 31.5; 95\% confidence interval, $12.8-77.2 ; P<.001)$. C-index of the prediction model was 0.74 .

Conclusions: The overall rate of dual antiplatelet therapy use in patients with acute coronary syndrome who underwent coronary artery bypass grafting was low and variable among surgeons. The use or nonuse was guided by previously established risk factors of recurrent ischemia and bleeding, along with surgeon preference. (J Thorac Cardiovasc Surg 2018;155:632-8)

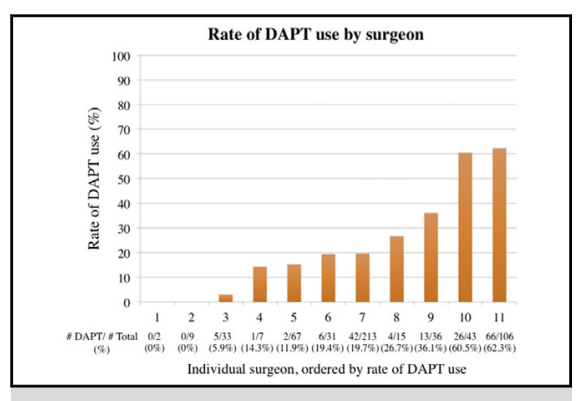

Variability of DAPT use after CABG among surgeons.

\section{Central Message}

Rate of DAPT use in patients with ACS who underwent $\mathrm{CABG}$ was low and variable among surgeons. The use or nonuse was guided by previously established risk factors of recurrent ischemia and bleeding.

\section{Perspective}

Resumption of DAPT after CABG in patients presenting with ACS is recommended, but the current practice pattern in the United States is unknown. Our retrospective review identified a significant variability in DAPT use among surgeons, and the use was guided by known risk factors of recurrent ischemia and bleeding.

See Editorial Commentary page 639.
Dual antiplatelet therapy (DAPT) in patients presenting with acute coronary syndrome (ACS) reduces ischemic events and improves long-term survival. ${ }^{1,2}$ Its benefit has been demonstrated in a subgroup of patients treated

From the ${ }^{\text {a Section }}$ of Cardiac Surgery, Yale University School of Medicine; and ${ }^{b}$ Department of Epidemiology of Microbial Diseases, Yale School of Public Health, New Haven, Conn.

Institutional Review Board: HIC \#1610018501, approval date November 1, 2016.

Received for publication May 13, 2017; revisions received Aug 28, 2017; accepted for publication Sept 19, 2017; available ahead of print Oct 19, 2017.

Address for reprints: Arnar Geirsson, MD, Section of Cardiac Surgery, Yale University School of Medicine, BB204, 330 Cedar St, PO Box 208039, New Haven, CT 06520 (E-mail: arnar.geirsson@yale.edu).

0022-5223/\$0.00

Published by Elsevier Inc. on behalf of The American Association for Thoracic Surgery

https://doi.org/10.1016/j.jtcvs.2017.09.092 with percutaneous coronary intervention (PCI) after presentation with ACS. Resumption of DAPT after coronary artery bypass grafting (CABG) in patients presenting with ACS is a class I recommendation in 2016 American College of Cardiology/American Heart Association guideline-focused updates, 3 "Strong Recommendation" in 2012 Canadian Cardiovascular Society guideline, ${ }^{4}$ and class IIa recommendation in 2014 European Society of Cardiology/European Association for

- Scanning this $\mathrm{QR}$ code will take you to a supplemental video for the article. 

Abbreviations and Acronyms
ACS = acute coronary syndrome
$\mathrm{ADP}=$ adenosine diphosphate
$\mathrm{CABG}=$ coronary artery bypass grafting
CI = confidence interval
DAPT $=$ dual antiplatelet therapy
MACCE $=$ major adverse cardiac and cerebrovascular events
MI = myocardial infarction
$\mathrm{OR} \quad=$ odds ratio
PCI = percutaneous coronary intervention

Cardio-Thoracic Surgery. ${ }^{5}$ Despite these current recommendations, there remains a lack of robust data to support DAPT use post-CABG.

A recent survey of Canadian cardiac surgeons demonstrated significant surgeon-to-surgeon variations in the rate of DAPT use after CABG, the level of awareness of current guidelines, and beliefs toward the potential benefit of DAPT. ${ }^{6}$ Although the Canadian data are likely a reasonable estimate of the practice pattern, knowledge, and attitudes of the US cardiac surgeons, there are no data to demonstrate a practice pattern at US institutions. Despite the recommendations, the expected benefits derived from the reduction of ischemia with DAPT must be balanced against the increased risk of bleeding, which may partly explain the nonroutine DAPT use that is perhaps more pronounced in the surgical population. A clinical score system to guide the prolonged use of DAPT based on the balance between predicted risk of bleeding and benefit of ischemia has been derived and validated using a multinational trial registry, ${ }^{7}$ and such a score may aid in a risk-adjusted evaluation of DAPT use after CABG. In this study, we aimed to investigate the practice pattern of cardiac surgeons at our institution regarding the use of DAPT by following patients with ACS undergoing CABG to assess (1) whether the current DAPT use pattern is based on the clinical principles of risk-benefit balance of bleeding and ischemia and (2) whether a prediction model for DAPT use with adequate discriminatory ability can be constructed.

\section{MATERIALS AND METHODS \\ Patient Population}

We conducted a retrospective review of consecutive adult patients who underwent CABG between July 2014 and September 2016 at Yale New Haven Hospital. Both isolated and concomitant CABG were included. This initial cohort consisted of 1084 patients. We excluded patients who died before discharge $(n=19)$; patients who presented with stable angina, no symptoms, or no evidence of recent myocardial infarction (MI) $(\mathrm{n}=494)$; and patients with missing DAPT data $(\mathrm{n}=16)$. The remaining cohort of patients consisted of 572 patients who presented with ACS (unstable angina, non-ST-elevation MI, or ST-elevation MI) who underwent isolated or concomitant CABG at our institution. Although the guideline states that resumption, rather than initiation of DAPT, is recommended after $\mathrm{CABG},{ }^{3}$ we elected to include all patients presenting with ACS regardless of preoperative DAPT use because DAPT use is recommended for all patients with ACS outside of contraindications, and the risk-benefit balance of recurrent ischemia and bleeding that guides the DAPT use is expected to be modulated by various intraoperative and postoperative factors.

The Yale Institutional Review Board approved the study, and individual patient consent was waived. The cohort was then divided into patients who were discharged on DAPT and patients who were not discharged on DAPT to undergo comparisons of baseline, operative, and postoperative characteristics. The primary outcome of this study was hospital discharge from the index hospitalization with DAPT.

\section{Collected Data}

Baseline demographic data, outlined in Table 1, and operative and postoperative data, outlined in Table 2, were collected. The Society of Thoracic Surgeons data definitions (version 2.81) were used in all data fields. Data were collected up to postoperative day 30 or to the time of hospital discharge, whichever was longer. The specific type of the second antiplatelet therapy was not defined. Ventricular function was determined by echocardiography using subjective assessment, Simpson's method, or triplane 3-dimensional reconstruction in multiple views by attending cardiologists.

\section{Statistical Analysis}

Differences in patient characteristics were compared with 2-tailed $t$ test, chi-square test, or Fisher exact test, where appropriate. Continuous variables are expressed in median with interquartile range $(\mathrm{Q} 1, \mathrm{Q} 3)$ format unless otherwise specified. In the tables, categoric variables are expressed in row percentages. Fixed-effect logistic regression model was constructed with input variables consisting of variables outlined in Tables 1 and 2, with the exception of crossclamp time and cardiopulmonary bypass time, because these 2 variables showed significant collinearity with cardiopulmonary bypass use, which is a clinically plausible correlation. In addition, dummy variables specifying surgeons who conducted each operation were included in the model. To account for surgeon-specific preference for DAPT use, mixed-effect model, treating surgeon variable as a random effect, was also constructed. By using the fixed-effect model, 5 -fold cross-validation was conducted to generate 5 sets of randomly sampled 80:20 training and validation cohorts to evaluate sensitivity and specificity across a range of thresholds. Means of sensitivities and specificities were used to construct a receiver operating characteristics curve, and a c-index was calculated to evaluate its ability to predict discharge on DAPT. Mixed-effect model was fit in R 3.3.2 using the lme4 package. $^{8}$ All other analysis was conducted with SAS 9.4 (SAS Institute Inc, Cary, NC).

\section{RESULTS}

After the exclusion outlined in the "Materials and Methods" section, we identified 572 consecutive patients who presented with ACS and subsequently underwent isolated or concomitant CABG. Of these patients, 165 $(29 \%)$ were discharged from the hospital on DAPT after the index hospitalization. Baseline demographics and patient characteristics are outlined in Table 1. The following variables differed significantly between patients who were and were not discharged on DAPT using univariate analysis: age (63 vs 67 years, $P<.001)$, history of stroke $(10.9 \%$ vs $5.4 \%, P=.03)$, previous PCI $(42.4 \%$ vs $32.7 \%, P=.03)$, adenosine diphosphate (ADP) inhibitor use before CABG 
TABLE 1. Baseline characteristics of patients who underwent isolated/concomitant coronary artery bypass grafting

\begin{tabular}{|c|c|c|c|}
\hline Variables & DAPT at discharge $(n=165)$ & No DAPT at discharge $(n=407)$ & $P$ value \\
\hline Age, $y$ & $63(57,69)$ & $67(60.5,74)$ & $<.0001$ \\
\hline Female & $53(34.9 \%)$ & $99(65.1 \%)$ & .06 \\
\hline \multicolumn{4}{|l|}{ Race } \\
\hline White & $134(27.7 \%)$ & $349(72.3 \%)$ & .26 \\
\hline African-American & $10(41.7 \%)$ & $14(58.3 \%)$ & \\
\hline Other & $21(32.3 \%)$ & $44(67.7 \%)$ & \\
\hline Body mass index $\left(\mathrm{kg} / \mathrm{m}^{2}\right)$ & $28.6(25.5,32.6)$ & $28.8(25.6,33.5)$ & .98 \\
\hline \multicolumn{4}{|l|}{ ACS type } \\
\hline Unstable angina & $89(29.9 \%)$ & $209(70.1 \%)$ & .76 \\
\hline NSTEMI & $59(27.1 \%)$ & $159(72.9 \%)$ & \\
\hline STEMI & $17(30.4 \%)$ & $39(69.6 \%)$ & \\
\hline \multicolumn{4}{|l|}{ Baseline comorbidities } \\
\hline Diabetes & $89(31.7 \%)$ & $192(68.3 \%)$ & .17 \\
\hline Dyslipidemia & $151(29.1 \%)$ & $368(70.9 \%)$ & .87 \\
\hline Dialysis & $2(16.7 \%)$ & $10(83.3 \%)$ & .52 \\
\hline Preoperative creatinine $(\mathrm{mg} / \mathrm{dL})$ & $0.9(0.8,1.1)$ & $0.9(0.8,1.2)$ & .38 \\
\hline Moderate-severe chronic lung disease & $3(25.0 \%)$ & $9(75.0 \%)$ & 1.0 \\
\hline Liver disease & $7(38.9 \%)$ & $11(61.1 \%)$ & .42 \\
\hline Peripheral vascular disease & $24(29.3 \%)$ & $58(70.7 \%)$ & .89 \\
\hline Stroke & $18(45.0 \%)$ & $22(55.0 \%)$ & .03 \\
\hline MI & $113(28.3 \%)$ & $286(71.7 \%)$ & .69 \\
\hline Cardiogenic shock & $11(40.7 \%)$ & $16(59.3 \%)$ & .19 \\
\hline Previous CABG & $3(18.8 \%)$ & $13(81.2 \%)$ & .57 \\
\hline Previous PCI & $70(34.5 \%)$ & $133(65.5 \%)$ & .03 \\
\hline ADP inhibitor within $5 \mathrm{~d}$ of $\mathrm{CABG}$ & $58(40.9 \%)$ & $84(59.1 \%)$ & .0002 \\
\hline ASA within $5 \mathrm{~d}$ of $\mathrm{CABG}$ & $137(29.3 \%)$ & $330(70.7 \%)$ & .81 \\
\hline No. of diseased vessels & $3(2,3)$ & $3(3,3)$ & .11 \\
\hline Preoperative ejection fraction $(\%)$ & $55(41,61)$ & $55(43,60)$ & .82 \\
\hline Preoperative platelet count (thousands) & $222(188,289)$ & $215(179,256)$ & .006 \\
\hline
\end{tabular}

Shown is number of patients $(\mathrm{n})$ and row percentage for categoric variables and median $(\mathrm{Q} 1, \mathrm{Q} 3)$ for continuous variables. Bold denotes $P<.05 . D A P T$, Dual antiplatelet therapy; $A C S$, acute coronary syndrome; NSTEMI, non-ST-elevation myocardial infarction; STEMI, ST-elevation myocardial infarction; $M I$, myocardial infarction; $C A B G$, coronary artery bypass grafting; $P C I$, percutaneous coronary intervention; $A D P$, adenosine diphosphate; $A S A$, acetylsalicylic acid.

$(35.2 \%$ vs $20.7 \%, P<.01)$, and preoperative platelet counts $(222[188,289]$ vs $215[179,256]$ in thousands, $P<.01)$.

Operative variables and postoperative outcomes are outlined in Table 2. On univariate analysis, patients who were and were not discharged on DAPT had statistically significant differences in the following variables: concomitant CABG $(6.7 \%$ vs $14.7 \%, P<.01)$, off-pump CABG (52.7\% vs $4.4 \%, P<.001)$, and hospital discharge on oral anticoagulant $(12.5 \%$ vs $18.9 \%, P<.001)$. Of note, postoperative stroke, renal failure, and take back for bleeding were rare and did not differ significantly between the groups. The rates of patients discharged on DAPT by surgeon are demonstrated in Figure 1 and ranged between $0 \%$ and $62.3 \%$.

The result of fixed-effect multivariate logistic regression is outlined in Table 3. Odds ratio (OR) greater than 1.0 indicates that the variable is associated with increased odds of being discharged with DAPT. Increase in age by 1 year was associated with lower odds of being discharged with DAPT (OR, 0.95; 95\% confidence interval [CI],
0.92-0.98, $P=.001$ ). Likewise, patients who were on oral anticoagulant at discharge were less likely to be discharged on DAPT (OR, $0.2 ; 95 \%$ CI, 0.06-0.6; $P=.004$ ). Patients who were administered an ADP inhibitor within 5 days before surgery were more likely to be discharged on DAPT (OR, 3.0; 95\% CI, 1.6-5.8; $P<.001$ ). Patients undergoing off-pump CABG compared with on-pump CABG were significantly more likely to be discharged on DAPT (OR, 33.5; 95\% CI, 12.5-100.5; $P<.001$ ). Compared with the patients operated on by the surgeon with the lowest rate of DAPT use, patients operated on by all other surgeons had increased odds of being discharged on DAPT, with the OR ranging from 7.6 to 28.7 in each compared surgeon (all $P<.05$ ).

The result of mixed-effect multivariate logistic regression is outlined in Table 4. In this model, surgeon variable was treated as a random effect, and thus the model accounts for individual surgeon's preference for DAPT use. Patients operated on by 2 surgeons with no DAPT use (surgeons 1 and 2 in Figure 1) were excluded (total $\mathrm{n}=11$ ). 
TABLE 2. Operative variables and postoperative outcomes

\begin{tabular}{|c|c|c|c|}
\hline Variables & DAPT at discharge $(n=165)$ & No DAPT at discharge $(n=407)$ & $P$ value \\
\hline \multicolumn{4}{|l|}{ Operation status } \\
\hline Elective & $29(29.6 \%)$ & $69(70.4 \%)$ & .50 \\
\hline Urgent & $126(28.1 \%)$ & $322(71.9 \%)$ & \\
\hline Emergency & $10(38.5 \%)$ & $16(61.5 \%)$ & \\
\hline \multicolumn{4}{|l|}{ Surgery } \\
\hline Isolated CABG & $154(30.7 \%)$ & $347(69.3 \%)$ & .008 \\
\hline Concomitant CABG & $11(15.5 \%)$ & $60(84.5 \%)$ & \\
\hline Mitral valve procedure & $14(87.5 \%)$ & $2(12.5 \%)$ & .17 \\
\hline Aortic valve procedure & $25(92.6 \%)$ & $2(7.4 \%)$ & .009 \\
\hline Off-pump CABG & $87(82.9 \%)$ & $18(17.1 \%)$ & $<.0001$ \\
\hline CPB time, $\min$ & $94(80,111)$ & $91(76,113)$ & .92 \\
\hline Crossclamp time, min & $69(55.5,83)$ & $65(53,83)$ & .95 \\
\hline No. of arterial distal anastomoses & $1(1,1)$ & $1(1,1)$ & .19 \\
\hline No. of venous distal anastomoses & $2(1,2)$ & $2(1,2)$ & .38 \\
\hline IABP use & $25(32.5 \%)$ & $52(67.5 \%)$ & .50 \\
\hline ICU length, $h$ & $62.8(46,89)$ & $69(47.2,93)$ & .24 \\
\hline Take back for bleeding & $0(0 \%)$ & $5(100.0 \%)$ & .33 \\
\hline Stroke & $3(27.3 \%)$ & $8(72.7 \%)$ & .73 \\
\hline Renal failure & $3(50.0 \%)$ & $3(50.0 \%)$ & .15 \\
\hline Postoperative transfusion (any blood product) & $55(27.5 \%)$ & $145(72.5 \%)$ & .63 \\
\hline Discharge on warfarin/DTI/Xa inhibitor & $11(12.5 \%)$ & $77(87.5 \%)$ & .0002 \\
\hline
\end{tabular}

Shown is number of patient (n) and row percentage for categoric variables and median (Q1, Q3) for continuous variables. Bold denotes $P<.05$. DAPT, Dual antiplatelet therapy; $C A B G$, coronary artery bypass grafting; $C P B$, cardiopulmonary bypass; $I A B P$, intra-aortic balloon pump; $I C U$, intensive care unit; $D T I$, direct thrombin inhibitor.

Age, ADP inhibitor use within 5 days before the operation, off-pump CABG, and anticoagulant use at discharge were all significantly associated with DAPT use, with the OR similar to the OR demonstrated in the fixed-effect model. In this model, preoperative dialysis was also significantly associated with lower odds of DAPT use (OR, 0.07; 95\%

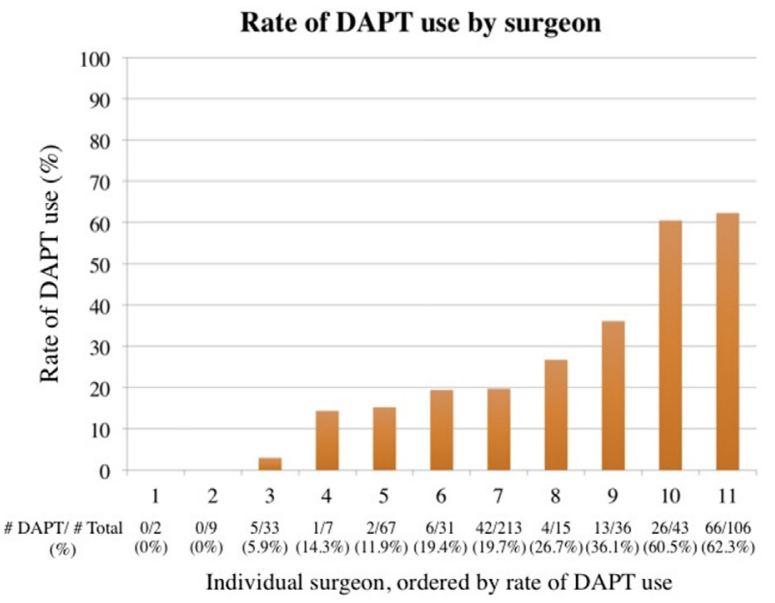

FIGURE 1. Rate of DAPT use by surgeon. Rates of DAPT use by individual surgeon. \#DAPT/\#Total represents the number of patients discharged on DAPT over the total number of patients operated on by the particular surgeon. DAPT, Dual antiplatelet therapy.
CI, 0.01-0.94; $P=.045)$. A receiver operating characteristics analysis of the fixed-effect multivariate logistic regression yielded a c-index of 0.74 .

\section{DISCUSSION}

\section{Predictors of Dual Antiplatelet Therapy Use}

In this study, we identified that the rate of hospital discharge on DAPT was $29 \%$ in patients who presented with ACS and underwent CABG. Multivariate analysis demonstrated independent predictors of DAPT use after CABG: younger age, ADP inhibitor use within 5 days before surgery, surgeon preference, off-pump CABG, and patients not discharged on an oral anticoagulant. Advanced age is a well-defined risk factor for bleeding in patients who undergo and receive $\mathrm{DAPT}^{7,9}$; therefore, it is likely that the presumed risk of bleeding was the driving factor in the nonuse in patients with advanced age. The cause of increased risk of bleeding is likely multifactorial, including tissue fragility, comorbidity, and fall risk. Likewise, the use of oral anticoagulant in combination with DAPT substantiates the risk of bleeding. In a subgroup analysis of Randomized Evaluation of Long-Term Anticoagulation Therapy trial, ${ }^{10}$ the annual risk of major bleeding on warfarin in combination with DAPT was $6.3 \%$ compared with $2.8 \%$ in the cohort with 
TABLE 3. Fixed-effect multivariate logistic regression for dual antiplatelet therapy use

\begin{tabular}{lccc}
\hline \multicolumn{1}{c}{ Variables } & OR & $\mathbf{9 5 \% ~ C I ~}$ & $\begin{array}{c}\boldsymbol{P} \\
\text { value }\end{array}$ \\
\hline Age (per 1-y increase) & 0.95 & $0.92-0.98$ & $\mathbf{. 0 0 1}$ \\
ADP inhibitor use within 5 d & 3.03 & $1.59-5.84$ & $<.001$ \\
Off-pump CABG & 33.53 & $12.48-100.51$ & $<.001$ \\
Anticoagulant at discharge & 0.20 & $0.06-0.57$ & $\mathbf{. 0 0 4}$ \\
\hline Surgeon* & $7.57-28.72$ & - & $<.001-.04$ \\
\hline
\end{tabular}

Shown is the result of fixed-effect multivariate logistic regression for the odds of DAPT use. OR greater than 1 indicates increased odds of DAPT use. Bold denotes $P<.05$. $O R$, Odds ratio; $C I$, confidence interval; $A D P$, adenosine diphosphate; $C A B G$, coronary artery bypass grafting. *Individual surgeon, when compared with the surgeon with the lowest rate of use, was a statistically significant variable with the range of $\mathrm{ORs}$ and $P$ values outlined.

warfarin alone (hazard ratio, 2.34; 95\% CI, 1.53-3.57). These evidences argue for careful consideration of DAPT resumption in these patient populations and likely were the driving factors of increased nonuse.

In comparison, postoperative use of DAPT after off-pump CABG remains controversial. Current evidence suggests that a transient hypercoagulable state occurs after off-pump CABG and that this population may derive benefit from DAPT. ${ }^{11}$ Prevention of Coronary arteRY bypaSS occlusion after off-pump procedures, a single-center trial, randomized 300 patients into aspirin monotherapy or DAPT after off-pump CABG. ${ }^{12}$ Although the sample size in this trial was powered to identify the difference in platelet response, the study demonstrated superior vein graft patency in the DAPT cohort at 12 months after the surgery. However, there is no randomized controlled trial that is powered sufficiently to assess the effect of DAPT on major adverse cardiac and cerebrovascular events (MACCE) after off-pump or on-pump CABG.

\section{Dual Antiplatelet Therapy Use After Acute Coronary Syndrome and Coronary Artery Bypass Grafting}

In the cohort of patients presenting with ACS with and without subsequent PCI, large clinical trials have demonstrated a significant clinical benefit of DAPT compared

TABLE 4. Mixed-effect multivariate logistic regression for dual antiplatelet therapy use

\begin{tabular}{lccr}
\hline \multicolumn{1}{c}{ Variables } & OR & $\mathbf{9 5} \%$ CI & $\boldsymbol{P}$ value \\
\hline Age (per 1-y increase) & 0.95 & $0.92-0.98$ & $<. \mathbf{0 0 1}$ \\
ADP inhibitor use within 5 d & 2.92 & $1.62-5.24$ & $<.001$ \\
Preoperative dialysis & 0.07 & $0.01-0.94$ & $\mathbf{. 0 4 5}$ \\
Off-pump CABG & 31.47 & $12.83-77.22$ & $<. \mathbf{0 0 1}$ \\
Anticoagulant at discharge & 0.20 & $0.07-0.55$ & $\mathbf{. 0 0 2}$ \\
\hline
\end{tabular}

Shown is the result of mixed-effect multivariate logistic regression for the odds of DAPT use. Surgeon variable is treated as a random effect. OR greater than 1 indicates increased odds of DAPT use. Bold denotes $P<.05$. OR, Odds ratio; $C I$, confidence interval; $A D P$, adenosine diphosphate; $C A B G$, coronary artery bypass grafting. with aspirin monotherapy in the secondary prevention of death, MI, stroke, and the need for revascularization. ${ }^{1,2,13}$ On the basis of this evidence, DAPT is recommended for patients presenting with ACS. In comparison, current evidence on the use of DAPT after CABG in patients presenting with ACS is limited, although resumption of DAPT after CABG is a class I recommendation in the 2016 American Heart Association/American College of Cardiology guideline. ${ }^{3}$ The best existing evidence evaluating the effect of DAPT on MACCE after CABG is limited to subgroup analyses of trials that were originally designed to evaluate the use of DAPT in ACS. In the cohort of the Clopidogrel in Unstable angina to prevent Recurrent ischemic Events trial, $16.5 \%$ of patients underwent CABG. In this subgroup of patients who underwent CABG, there was no statistically significant difference in the rate of MACCE in patients receiving DAPT compared with aspirin monotherapy (relative risk of $0.89 ; 95 \% \mathrm{CI}$, 0.71-1.11), although a trend toward risk reduction was observed. Risk of bleeding did not differ significantly between the monotherapy and DAPT cohorts in this CABG subgroup (relative risk, 1.27; 95\% CI, 0.96-1.69; $P=.095) .{ }^{14}$ A meta-analysis that included a subgroup analyses of patients in Therapeutic Outcomes by Optimizing Platelet Inhibition with Prasugrel-Thrombolysis in Myocardial Infarction-38 and Platelet Inhibition and Patient Outcomes trials who underwent CABG indicates that the higher-intensity antiplatelet agents, prasugrel and ticagrelor, are associated with a reduction in the rate of MACCE compared with clopidogrel as the second antiplatelet agent. ${ }^{15}$ There are small single-center randomized controlled trials comparing aspirin monotherapy and DAPT with clopidogrel in patients who underwent CABG, with a follow-up of up to 12 months after surgery. ${ }^{12,16,17}$ None of the trials were powered to evaluate the effect of DAPT on MACCE but have demonstrated superior vein graft patency in the DAPT cohort. Perhaps contrary to these findings, a randomized controlled trial comparing aspirin monotherapy with DAPT in patients undergoing elective $\mathrm{CABG}$ did not find a difference in 1year angiographic graft patency or degree of intimal hyperplasia by intravascular ultrasound. ${ }^{18}$ The observed benefits of prasugrel and ticagrelor in the CABG subgroup of Therapeutic Outcomes by Optimizing Platelet Inhibition with Prasugrel-Thrombolysis in Myocardial Infarction-38 and Platelet Inhibition and Patient Outcomes remain untested in the absence of a trial designed to evaluate this in a CABG cohort.

\section{Variability in Current Acute Coronary Syndrome Use}

We demonstrated that DAPT use among 11 surgeons at our institution varied dramatically. Surgeon preference also persisted in the fixed-effect multivariate model, 
indicating that surgeon preference plays a significant role in determining whether the patients are discharged on DAPT. However, the mixed-effect model showed that other clinical predictors (age, anticoagulant use, off-pump CABG) continued to have significant associations with DAPT use after accounting for the surgeon preference. The variable use among surgeons was an expected finding given the existing evidence on cardiac surgeons' variability in the practice and attitude toward DAPT use. A recent survey of 75 Canadian cardiac surgeons, which represents approximately half of the Canadian cardiac surgical community, identified that only $44.6 \%$ would routinely initiate DAPT after $\mathrm{CABG}$ in patients presenting with ACS, and that only $60 \%$ were aware of the current recommendations of routine DAPT use in patients presenting with ACS. In addition, only $64 \%$ believed that the patients with ACS undergoing CABG would benefit from DAPT use, with the rest believing that the benefit of DAPT would be limited only to those treated medically or percutaneously. ${ }^{6}$ In the context of limited evidence, the significant variability among surgeons in their attitude toward routine DAPT use after CABG may be understandable. The practice variability on DAPT use was also demonstrated in the cohort of patients presenting with ACS, for whom robust evidence advocates DAPT use. In their analysis of a multinational Global Registry of Acute Coronary Events between 2003 and 2007, Rao and associates ${ }^{19}$ identified that only $57 \%$ to $66 \%$ of patients presenting with ACS received DAPT within 24 hours of hospital presentation.

\section{Dual Antiplatelet Therapy Use Based on Ischemia Bleeding Risks}

Complicating the assessment of guideline adherence on DAPT use, in addition to the paucity of evidence, is the patient population in whom the theoretic bleeding risk surpasses the benefit derived from the reduction of ischemic event. The recommendation for routine use of DAPT should not be applied to populations who are at an increased risk for major bleeding. However, the risk-benefit balance of ischemia reduction and bleeding is difficult to assess in the absence of a clinical tool based on a robust quantification and modeling. A DAPT score was recently derived from a large PCI registry to assess the risk-benefit balance. ${ }^{7}$ This model cannot be applied directly to patients undergoing $\mathrm{CABG}$ because the derivation cohort consisted of patients who underwent PCI, and some variables are specific to PCI. Our prediction model identified several variables that were associated with DAPT use and nonuse according to the factors associated with known risks of ischemia and bleeding. Namely, advanced age and discharge on oral anticoagulants were associated with reduced odds of DAPT use, and off-pump CABG was associated with increased odds. A mixed-effect model was used to isolate the surgeon preference from this model,

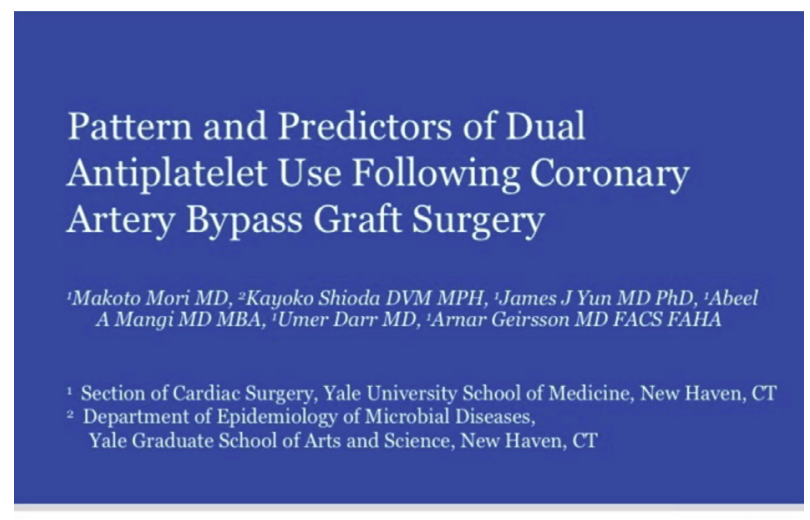

Yale SCHOOL OF MEDICINE

VIDEO 1. Brief summary of the study presented by first author Makoto Mori, MD. Video available at: http://www.jtcvsonline.org/article/S00225223(17)32133-5/fulltext.

and the congruence of OR of these variables between the mixed and fixed-effect model argues that the variables other than surgeon preference were also significantly associated with DAPT use or nonuse. The c-index of 0.74 argues for an adequate discriminatory ability of the model. Such a prediction model may aid in assessing the risk-adjusted evaluation of guideline adherence on DAPT therapy after CABG in a multicenter setting.

There remains a need for more robust evidence that supports or negates the proposed benefit of DAPT use after $\mathrm{CABG}$ in patients presenting with ACS. This would involve large clinical trials comparing aspirin monotherapy with DAPT, with clopidogrel, prasugrel, and ticagrelor in each arm as a second agent. In addition, studies on patients presumably at a higher risk of recurrent ischemia, such as those with renal failure, may aid in defining the role of DAPT in patients post-CABG. Until more evidence is available, the current guideline should be followed more rigorously.

\section{Study Limitations}

This is a single-center retrospective study and therefore harbors limitations inherent to such methodology and clinical setting. Long-term data on survival, bleeding, and recurrent ischemia were not included in the study because they were not the aims of this study. The test characteristics of the prediction model need to be further evaluated in a larger multicenter cohort.

\section{CONCLUSIONS}

At our center, $29 \%$ of patients who presented with ACS and underwent CABG were discharged on DAPT. Patients with increased age and on oral anticoagulant were less likely to receive DAPT, and patients who underwent off-pump CABG were more likely to receive DAPT. 
Surgeon preference was also a significant factor influencing the decision. The prediction model showed adequate test characteristics in discriminating the use or nonuse of DAPT on the basis of the risk factors of bleeding and recurrent ischemia (Video 1).

\section{Conflict of Interest Statement}

Authors have nothing to disclose with regard to commercial support.

\section{References}

1. Yusuf S, Zhao F, Mehta SR, Chrolavicius S, Tognoni G, Fox KK. Effects of clopidogrel in addition to aspirin in patients with acute coronary syndromes without ST-segment elevation. N Engl J Med. 2001;345:494-502.

2. Wallentin L, Becker RC, Budaj A, Cannon CP, Emanuelsson H, Held C, et al. Ticagrelor versus clopidogrel in patients with acute coronary syndromes. N Engl J Med. 2009;361:1045-57.

3. Levine GN, Bates ER, Bittl JA, Brindis RG, Fihn SD, Fleisher LA, et al. 2016 ACC/AHA Guideline Focused Update on Duration of Dual Antiplatelet Therapy in Patients With Coronary Artery Disease: A Report of the American College of Cardiology/American Heart Association Task Force on Clinical Practice Guidelines. Circulation. 2016;134:e123-55.

4. Tanguay JF, Bell AD, Ackman ML, Bauer RD, Cartier R, Chan WS, et al. Focused 2012 update of the Canadian Cardiovascular Society guidelines for the use of antiplatelet therapy. Can J Cardiol. 2013;29:1334-45.

5. Kolh P, Windecker S, Alfonso F, Collet JP, Cremer J, Falk V, et al. 2014 ESC/EACTS Guidelines on myocardial revascularization: the Task Force on Myocardial Revascularization of the European Society of Cardiology (ESC) and the European Association for Cardio-Thoracic Surgery (EACTS). Eur J Cardiothorac Surg. 2014;46:517-92.

6. Yanagawa B, Ruel M, Bonneau C, Lee MM, Chung J, Al Shouli S, et al. Dual antiplatelet therapy use by Canadian cardiac surgeons. J Thorac Cardiovasc Surg. 2015;150:1548-54.e3.

7. Yeh RW, Secemsky EA, Kereiakes DJ, Normand SL, Gershlick AH, Cohen DJ, et al. Development and validation of a prediction rule for benefit and harm of dual antiplatelet therapy beyond 1 year after percutaneous coronary intervention. JAm Med Assoc. 2016;315:1735-49.

8. Bates D, Mächler M, Bolker B, Walker S. Fitting linear mixed-effects models using lme4. J Stat Softw. 2015;67:48.

9. Mehta SK, Frutkin AD, Lindsey JB, House JA, Spertus JA, Rao SV, et al Bleeding in patients undergoing percutaneous coronary intervention: the development of a clinical risk algorithm from the National Cardiovascular Data Registry. Circ Cardiovasc Interv. 2009;2:222-9.

10. Dans AL, Connolly SJ, Wallentin L, Yang S, Nakamya J, Brueckmann M, et al. Concomitant use of antiplatelet therapy with dabigatran or warfarin in the Randomized Evaluation of Long-Term Anticoagulation Therapy (RE-LY) trial. Circulation. 2013;127:634-40.

11. Raja SG, Akhtar S. Hypercoagulable state after off-pump coronary artery bypass grafting: evidence, mechanisms and implications. Expert Rev Cardiovasc Ther. 2011:9:599-608.

12. Mannacio VA, Di Tommaso L, Antignan A, De Amicis V, Vosa C. Aspirin plus clopidogrel for optimal platelet inhibition following off-pump coronary artery bypass surgery: results from the CRYSSA (prevention of Coronary arteRY bypaSS occlusion After off-pump procedures) randomised study. Heart. 2012; 98:1710-5.

13. Wiviott SD, Braunwald E, McCabe CH, Montalescott G, Ruzyllo W, Gottlieb S, et al. Prasugrel versus clopidogrel in patients with acute coronary syndromes. N Engl J Med. 2007;357:2001-15.

14. Fox KA, Mehta SR, Peters R, Zhao F, Lakkis N, Gersh BJ, et al. Benefits and risks of the combination of clopidogrel and aspirin in patients undergoing surgical revascularization for non-ST-elevation acute coronary syndrome: the Clopidogrel in Unstable angina to prevent Recurrent ischemic Events (CURE) Trial. Circulation. 2004;110:1202-8.

15. Verma S, Goodman SG, Mehta SR, Latter DA, Ruel M, Gupta M, et al. Should dual antiplatelet therapy be used in patients following coronary artery bypass surgery? A meta-analysis of randomized controlled trials. BMC Surg. 2015;15: 112.

16. Gao G, Zheng Z, Pi Y, Lu B, Lu J, Hu S. Aspirin plus clopidogrel therapy increases early venous graft patency after coronary artery bypass surgery a single-center, randomized, controlled trial. J Am Coll Cardiol. 2010;56: $1639-43$.

17. Gasparovic H, Petricevic M, Kopjar T, Djuric Z, Svetina L, Biocina B. Impact of dual antiplatelet therapy on outcomes among aspirin-resistant patients following coronary artery bypass grafting. Am J Cardiol. 2014;113:1660-7.

18. Kulik A, Le May MR, Voisine P, Tardif JC, Delarochelliere R, Naidoo S, et al. Aspirin plus clopidogrel versus aspirin alone after coronary artery bypass grafting: the clopidogrel after surgery for coronary artery disease (CASCADE) Trial. Circulation. 2010;122:2680-7.

19. Rao RV, Goodman SG, Yan RT, Spencer FA, Fox KA, DeYoung JP, et al. Temporal trends and patterns of early clopidogrel use across the spectrum of acute coronary syndromes. Am Heart J. 2009;157:642-50.

Key Words: coronary artery bypass grafting, dual antiplatelet therapy, practice pattern 